\title{
Kernos
}

Revue internationale et pluridisciplinaire de religion grecque antique

19 | 2006

Varia

\section{Images, mythes, catalogues, généalogies et} mythographies

Présentation du colloque

\section{Lambros Couloubaritsis}

\section{(2) OpenEdition}

Journals

Édition électronique

URL : https://journals.openedition.org/kernos/423

DOI : $10.4000 /$ kernos.423

ISSN : 2034-7871

Éditeur

Centre international d'étude de la religion grecque antique

Édition imprimée

Date de publication : 1 janvier 2006

Pagination : 11-21

ISSN : 0776-3824

Référence électronique

Lambros Couloubaritsis, «Images, mythes, catalogues, généalogies et mythographies », Kernos [En

ligne], 19 | 2006, mis en ligne le 24 mai 2011, consulté le 24 août 2022. URL : http://

journals.openedition.org/kernos/423; DOI : https://doi.org/10.4000/kernos.423 


\title{
Images, mythes, catalogues, généalogies et mythographies Présentation du colloque
}

\author{
par Lambros COULOUBARITSIS
}

C'est la deuxième fois que l'Université Libre de Bruxelles prend en charge l'organisation d'un colloque du CIERGA sous ma direction ${ }^{1}$. Le thème de ce $\mathrm{X}^{\mathrm{e}}$ colloque, qui associe la question des catalogues et celle des généalogies, préoccupe depuis longtemps de nombreux membres du CIERGA et concerne un volet important des travaux du Centre de Philosophie ancienne de l'ULB depuis vingt-cinq ans, et plus récemment de son Séminaire interdisciplinaire de recherche et d'encadrement (SIRE), animé par Ioanna PapadopoulouBelmehdi. Il a été proposé par Claude Calame à l'occasion d'une discussion sur mon analyse des catalogues et des généalogies, lors du dernier colloque du réseau européen «Polymnia » sur la mythographie, dirigé par Jacqueline Fabre-Serris et Françoise Graziani. C'est la raison pour laquelle nous avons tenu à associer Claude Calame et le groupe «Polymnia » à cette manifestation, et organiser deux tables rondes parallèles de chercheurs appartenant aux deux groupes. Mais comme l'Université de Bruxelles est associée au réseau européen «Polymnia », il ne nous a pas semblé utile de séparer les deux séries d'interventions de l'ensemble des contributions ${ }^{2}$.

Je tiens néanmoins à remercier séparément mes collègues de l'ULB, et plus spécialement Michèle Broze, Baudouin Decharneux, Ioanna Papadopoulou, Françoise Labrique, Mihail Nasta et ceux, parmi les chercheurs du Centre, qui ont pris une part active à la réussite de cette manifestation. Depuis longtemps, chacun apporte sa pierre à l'élaboration de nos analyses des mythes et des rites à travers un projet commun et un esprit pluraliste et ouvert, débordant la littérature et la religion grecques.

Je souhaite, ensuite, associer à mes remerciements Claude Calame, Jacqueline Fabre-Serris, Françoise Graziani et Evangélos Moutsopoulos. Non seulement Claude Calame a inspiré une thématique qui nous est chère et accepté

\footnotetext{
${ }^{1}$ La précédente rencontre, qui était le quatrième colloque du CIERGA, a eu lieu en 1993 sur le thème : "Influences, emprunts et syncrétismes religieux en Grèce ancienne ». Les Actes ont été publiés dans Kernos 8 et 9 .

${ }^{2}$ Faisaient partie de la table ronde du SIRE, consacrée à la Polyvalence catalogique et généalogie: approches interdiciplinaires, les communications de I. Papadopoulou, M. Broze, A. Busine, S. Inowlocki, N. Massar, M. Nasta et G. Zographou de l'Univ. de Ioannina, avec comme intervenants : N. Brout, B. Decharneux, F. Labrique, V. Pirenne, A. Verbanck; faisaient partie du groupe Polymnia sur Formes génalogiques et catalogales dans la mythographie gréco-romaine, les communications de J. Fabre-Seiris, Fr. Graziani et E. Pellizer.
} 
d'associer son nom aux organisateurs du colloque, mais il n'a pas ménagé son temps pour réunir, avec un esprit critique et bienveillant, les thèmes qui ont été retenus. D'autre part, par leur collaboration, Jacqueline Fabre-Serris et Françoise Graziani témoignent de leur fidélité à nos échanges depuis plusieurs années. C'est aussi l'occasion de réitérer notre reconnaissance à Evangélos Moutsopoulos au moment où il quitte la présidence du CIERGA. Depuis la création de l'association, il a assumé cette tâche avec une tolérance exemplaire. Sa présence permanente et discrète a joué un rôle fécond dans son histoire et sa destinée.

Enfin, mes remerciements s'adressent plus spécialement à Vinciane Pirenne pour sa contribution à l'organisation du Colloque à un moment où j'étais très occupé par la publication de mon dernier ouvrage. Dans ces circonstances insolites, sa présence infatigable m'a réconforté, surtout pour la préparation de la première phase de l'organisation du colloque, sans doute la plus difficile. Je la remercie aussi de son efficacité incomparable dans cette nouvelle phase de publication des Actes dans Kernos.

Pour terminer, notre reconnaissance collective s'adresse aux institutions qui, en plus du CIERGA lui-même (toujours présent pour soutenir l'organisation des colloques en dépit de ses faibles moyens), ont apporté leur concours pour la réalisation de cette rencontre dans les meilleures conditions possibles : le Fonds National de la Recherche Scientifique de Belgique, la Faculté de Philosophie et Lettres de l'UlB et l'Institut des Hautes Études de Belgique.

Les communications, dont le nombre a été limité par des impératifs logistiques et économiques, forment un échantillon représentatif témoignant à la fois de l'ampleur de la question et des multiples ouvertures à de nouvelles recherches. Leur variété révèle la complexité du problème, et c'est pourquoi elles contribuent à une première mise au point sur une thématique, travaillée surtout en anthropologie, mais qui, en parallèle, préoccupe depuis longtemps les spécialistes de la culture antique.

Dans son introduction à la thématique du colloque, publiée ci-après, Claude Calame a esquissé d'une façon succincte et claire les axes de recherche que nous souhaitions aborder ou éprouver, sans exclure bien entendu d'autres perspectives. Dans les grandes lignes, il s'agissait d'aborder au moins trois situations différentes ou complémentaires: étude de données qui renvoient à l'horizon des procédés énumératifs; analyse de la différence entre pratiques des catalogues et généalogies; élucidation des rapports de ces pratiques avec la mythologie et la mythographie.

En présentant l'apport des différentes communications, je souhaite mettre en valeur les problèmes abordés, que résument les mots du titre, riches d'information : images, mythes, catalogues, généalogies et mythographies. L'ordre de succession de l'étude des textes, tout comme la mise en forme d'une table des matières ne sont jamais innocents. Ils reflètent une conception à la fois unitaire et plurielle à partir de laquelle s'organise une suite catalogique en vue 
d'établir des parentés et des filiations. Aussi ai-je choisi une présentation selon une typologie organisée à partir de deux positions extrêmes des pratiques énumératives: d'une part, les listes selon des séquences liées à des graphismes ou des images et, d'autre part, différentes formes narratives qui intègrent des procédés catalogiques. Entre ces deux extrêmes, j'ai réparti les discours catalogiques, en partant des listes (inventaires) et de leur transformation en catalogues jusqu'au rapport entre mythographie et mythologie, et en passant par deux pratiques éminentes des procédés catalogiques : les généalogies et les cheminements, qui utilisent des schèmes régulateurs, le premier étant celui de la «parenté », le second, celui du « chemin ».

Pour rendre plus explicite la cohérence des différents sujets, j’ai proposé un texte qui s'appuie sur mes propres recherches. Parmi les termes que j'ai analysés figure mythos, et sa différence avec legô et katalegô. Mais j'ai aussi abordé deux aspects du discours catalogique : celui qui utilise le schème de la parenté, mieux perçu généralement, et celui, plus implicite, régi par le schème du chemin, que j'ai illustré par l'épisode de Circé dans la dernière partie de l'Odyssée. Mon étude s'achève avec une actualisation de la question des catalogues et une esquisse de sa portée philosophique aujourd'hui. Cette ouverture vers l'avenir justifie sa place en conclusion de ces Actes.

Les communications exprimant deux situations extrêmes sont, d'une part, celles de Anne-Françoise Jaccottet, sur les généalogies et catalogues «sans paroles » à Olympie, et de Natacha Massar sur la «Chronique de Lindos », et, d'autre part, celle de Michel Briand, qui aborde le problème des catalogues et des résumés dans les romans grecs.

Parmi les procédés énumératifs, l'usage des listes est le plus clair. Il s'agit non seulement des listes d'éléments graphiques sur lesquelles s'est appuyé J. Goody pour analyser la différence entre oralité et écriture, mais également les images en série et autres objets servant, entre autres, aux rituels, avec les formulations qui s'y rapportent (dédicaces, dons, épiphanies, etc.) $)^{3}$, que l'on

\footnotetext{
3 Dans son exposé introductif au colloque, Cl. Calame illustre bien ce point, en s'appuyant sur les travaux de J. Goody (1977) et de Ch. Seydou (1989), en relevant l'évolution interprétative qui va du lien défendu par Goody entre écriture, archivisation et histoire, au lien préalable entre poésie orale selon deux fonctions de listes, analysées par Seydou comme des catalogues conformes à un trait sémantique commun et des inventaires d'objets hétérogènes dans un ensemble homogène. Je me permets d'ajouter que cette thématique peut intégrer les pratiques de la pictographie, considérées comme un obstacle à la multiplication des mots et à la mise en ordre des signes (I.J. GelB, Théorie de l'écriture, Paris, Flammarion, 1958 [or. angl. 1952], et J. DefraNCIS, Visible Speech. The Diverse Oneness of Writing, Hawai University Press, 1990). C'est la raison pour laquelle J. Goody et J. Watt ont accordé une autonomie à la parole par rapport aux supports matériels (donc aussi aux images), comprenant les sociétés sans écriture où règnerait une circulation libre et instable de l'information, en dehors d'une hiérarchisation du savoir et d'une mémoire culturelle efficace (J. GOODY, The Intertface Between the Oral and the Written, Cambridge Univ. Press, 1987, et avec J. WATT, Literacy in Traditional Societies, Cambridge Univ. Press, 1968). Or, à l'instar de Ch. Seydoux qui dégage des procédés catalogiques chez les Peuls du Niger, C. Severi établit l'existence chez les Cuna d'Amérique de savoirs pictographiques en forme de listes et de catalogues (C. SEVERI, "La mémoire rituelle - Expérience, tradition, historicité », in A. MONOD et A. Molinié (dir.), Mémoires de la tradition, Paris, Société d'Ethnologie-Nan-
} 
trouve dans l'espace collectif de la cité (agoras, sanctuaires, gymnases, nécropoles... $)^{4}$. Les communications de Anne-Françoise Jaccottet et de Natacha Massar montrent comment il est possible de faire voir, à partir de ces genres de listes, associées à des objets et des lieux religieux (en l'occurrence les sanctuaires d'Olympie et de Lindos), les liens avec les données de la culture de l'époque, voire avec l'espace civil. Il apparaît que la topologie religieuse ne peut être tributaire de la mythologie et d'autres variables culturelles, que révèlent les pratiques séquentielles, comme les séries graphiques, les catalogues et les généalogies. Dès lors qu'il y a des images porteuses d'informations mythologiques et généalogiques, une forme de mythographie sans parole devient possible autour d'une koinè mythologique et culturelle. Il arrive même que s'accomplisse, par ce biais, une consolidation de l'image de marque d'un lieu ou d'un édifice religieux ou public. Bref, les signes graphiques et les images recèlent à la fois une masse d'informations qu'on peut déployer par l'imagination ou la pensée, et une force symbolique qui touche au statut des convictions et de l'affectivité.

À l'autre bout, là où le katalegein et les catalogues sont utilisés, là où domine le champ de l'oralité qui dit les choses successivement, qui décrit et raconte conformément à un ordre, le discours met en ouvre plus explicitement une façon de dire les choses qui est le sens premier du mot mythos, avant que ce terme ne fusionne avec le logos pour exprimer aussi le récit et le fait de raconter. C'est cette dernière (con)fusion, déjà présente chez Platon, qui a été abordée par la communication de Michel Briand, qui témoigne des multiples transmutations de la culture antique à une période plus tardive.

M. Briand étudie cinq romans (mythoi), écrits par Xénophon d’Éphèse, Chariton, Achille Tatius, Longus et Héliodore, et montre que l'on y trouve différentes pratiques de la mythologie sous forme catalogique. Cela suppose qu'en plus des romans habituels dominés par la diègèsis (Daphnis et Chloè, Leucippè et Clitophon) il existe des romans utilisant des discours catalogiques ou encore qui intègrent des mythoi (contes, fables, récits...) en catalogues (Chaireas et Callirohè, Éthiopiques et les Éphésiaques). Cela suppose certes

terre, 1993; «Penser par séquences, penser par territoires - Cosmologie et art de la mémoire dans la pictographie des Indiens Cuna », Communications, 41 [1985], p. 169-190; " Paroles durables, écritures perdues. Réflexions sur la pictographie cuna », in M. DEtIENnE (dir.), Transcrire les mythologies, Paris, Albin Michel, p. 45-72, n. 226-232). Tout cela montre que la parole peut être soutenue par autre chose que par le signe. Comme le montrent les communications de A.-F. Jaccottet et de N. Massar, cette autre chose peut être de l'ordre de l'image (l'iconographie devenant ainsi de la mythographie), d'œuvres d'art ou de cultes véhiculant une sorte de mythographie sans image, riche d'information.

${ }^{4}$ C'est un point que Annie Verbanck a relevé lors de la discussion, en indiquant que l'accumulation de ces données (images, objets...) organise l'identité du groupe et de la cité, modèle de réflexion sur le passé, tout en assurant au présent un contexte commun, structuré et signifiant. L'étude de l'iconographie se déployant sur le mode de la série (panthéons, épisodes héroïques, processions, épreuves sportives, représentations des mois et des saisons, etc.) éclaire l'organisation sociale et politique. Loin d'illustrer simplement des récits, « le travail figuré serait une construction autonome de l'imaginaire, qui génère puis diffuse un substrat visuel indispensable, où le mythe puise fonction et fascination ». 
une modification du langage, où le terme «mythe » élargit ses usages, intégrant indifféremment le récit et la fiction, voire le logos plurivoque jusqu'à son usage extrême sur le mode catalogique. C'est ce genre de (con)fusion qui poussa Marcel Detienne à nier qu'il existait un genre que l'on puisse qualifier de "mythe », ne serait-ce que parce que, parmi toutes les formes narratives, aucune ne peut prétendre à ce privilège 5 .

Ioanna Papadopoulou approfondit le lien que j’ai esquissé entre les éléments homériques et le caractère générique du katalegô, tout en intégrant d'autres pratiques catalogiques que celle de la parenté. Ce lien est d'autant plus intéressant qu'il concerne Hérodote, lequel est l'un des premiers à écarter la sémantique du mythos de sa pensée, pour faire prévaloir à la fois l'élément émotionnel dans le logos relatant les choses merveilleuses (thaumasia) et ses prétentions argumentatives. Hérodote substitue aux Muses et à l'inspiration surnaturelle « la parole contemporaine des logo $i$ », sans néanmoins prétendre dénombrer, par exemple, une armée comme celle de Xerxès. Les généalogies qu'il mentionne sont soumises à son projet argumentatif. En allant d'Homère à Hérodote, I. Papadopoulou évoque le débat sur l'existence ou non des chroniques prélittéraires, et attire l'attention sur le fait qu'un procédé catalogique proche d'Hésiode a dominé avant et autour d'Hérodote, mais que celui-ci l'a modifié en faveur d'un style mixte inspiré d'Homère, d'un style où la démarche mimétique intègre davantage l'émotion et la construction argumentative. Elle relève aussi une attitude commune, critique et sélective, à l'égard des généalogies chez Homère et chez Hérodote. Dès lors, non seulement l'usage de katalegein est abondant chez Hérodote, en y intégrant également les pratiques généalogiques et les espaces géographiques, mais le logos trouve de nouvelles destinées. Le long inventaire des contingents de l'armée de Xerxès illustre la transformation intergénérique qui s'opère grâce aux références symboliques à l'Iliade, aux mises en scène en spectacle et aux promotions d'éléments dramatiques. Le chemin est ainsi ouvert à une multiplicité de pratiques catalogiques.

La communication de Gerasimoula Zographou (La généalogie des rois de Sparte : le cas de Démarate (Hérodote VI, 51-70) ${ }^{6}$, qui nous rapproche déjà de la problématique généalogique, illustre bien ce qui vient d'être dit. C'est à l'occasion des séquences relatant l'expédition perse qui menace la Grèce (discours catalogique utilisant le schème du chemin) que fait irruption la généalogie des rois afin de départager la légitimité du pouvoir. Il apparaît que Démarate, étant davantage tributaire d'un passé religieux et généalogiquement proche d'Hélène et d'Hercule, avait un droit héréditaire sur le trône supérieur à celui de Cléomène. Cependant les ambiguités de sa généalogie ont été exploitées par ce dernier qui s'adressa, mais sans succès, à l'oracle de Delphes. Nous découvrons que, non seulement une généalogie peut faire irrup-

\footnotetext{
5 M. DetiEnNe, L'invention de la mythologie, Paris, Gallimard, 1981, qui s'inspire des travaux de l'africaniste P. SMITH, Le récit populaire au Rwanda, Paris, 1975.

${ }^{6}$ Le texte de G. Zographou sera publié dans le prochain volume de $\operatorname{Kernos}(20,2007)$.
} 
tion via un récit (perspective catalogique), mais que ce récit peut associer politique et religion illustrant l'axe mythologique, voire rituélique ${ }^{7}$.

Michèle Broze, Aude Busine et Sabrina Inowlocki mettent davantage encore en évidence la complexité de la structure catalogique, en montrant que les catalogues des sages ont été intégrés dans les démarches philosophiques et apologétiques des Hellènes, des juifs et des chrétiens, et constitués à partir de différents critères (renommée, religion, science, magie, divination, philosophie, politique). Leur fonctionnalité est d'inclure ou d'exclure des peuples, ce qui leur confère tantôt un rôle unificateur, voire d'appropriation de l'auteur, tantôt un rôle de discrimination, voire de rejet. Cette conjonction entre démarche profusionnelle et variablement référentielle permet d'organiser la transmission des savoirs et les débats interculturels et religieux. À cette structure synchronique, il convient de lier également une lecture diachronique qui légitime les références, en remontant au passé, grâce aux pratiques généalogiques et à l'extension dans l'espace à travers les voyages, avec comme référence la culture chaldaïque. Pareille analyse révèle la pluridimensionnalité de la pratique des catalogues.

Dans un sens plus concret, Martin Steinrück s'appuie sur les Vies de philosophes et de sophistes d'Eunape pour montrer que les listes qui alignent des objets ou des personnes ne se limitent pas à un répertoire; elles glissent vers une forme narrative bouclée, mais cachée, qui marque la spécificité de la forme catalogique. Le processus de successions atteste une sorte de « généalogie » philosophique qui départage les filiations philosophiques de telle sorte que, sur le modèle des Éhées du Catalogue de femmes pseudo-hésiodique, il produit une histoire héroïque du néoplatonisme, qui doit conduire, par une répétition de l'âge de fer, à un nouveau combat contre les destructeurs de l'hellénisme.

La question de la transmission du savoir a été également abordée par Charles Delattre, mais sous l'éclairage de la doxographie. Tandis que la mythographie utilise un enchaînement d'énoncés servant à relater les hauts faits de personnages remontant à l'âge héroïque, la doxographie fait succéder les opinions des philosophes et des intellectuels, au point de les opposer, d'une façon erronée, selon le mythe et l'histoire. Or, ces procédés catalogiques ne seraient que deux dispositifs proches échangeant leurs modèles narratifs, selon une structure où se succèdent métaphores, catalogues et diagrammes (Dumézil). Cela met en jeu les influences au moyen de métaphores généalogiques (filiation), géométriques (ligne, intersection...), botaniques (se greffer...), par le tissage (fils, trame...). Modèle dominant chez Diogène Laërce, la filiation métaphorique organise et hiérarchise le monde philosophi-

\footnotetext{
${ }^{7}$ Comme je l'ai montré dans deux communications qui intègrent le rite dans la question des catalogues, l'axe mythologique de la structure du «mythe » correspond à un axe opérationnel, lequel inclut des cérémonies, des hymnes, des prières, des sacrifices, des sacrements, des transes et envoûtements, etc. $C f$. « Le discours catalogique et la constitution du monde des esprits », in $L e$ monde des esprits (Actes du colloque de l'ULB, novembre 2003), et "Mythe et rites », in Oralitéécriture: les rites (Actes du colloque de Mons, novembre 2006), à paraître en 2007.
} 
que (par les successions). Selon l'auteur, elle est encore utilisée, alors qu'elle serait la moins apte à traiter la transmission du savoir.

Ces approches fondées sur les catalogues révèlent que, selon les perspectives retenues, la généalogie se tient à proximité des pratiques catalogiques, tantôt d'une façon directe et tantôt d'une façon métaphorique. Elle se soumet à la pratique catalogique soit comme une de ses parties, soit comme l'une de ses orientations une fois qu'elle l'investit par le schème de la parenté, soit encore sur le mode métaphorique; elle apparaît ainsi comme une des espèces du discours catalogique, lequel ouvre à d'autres applications par d'autres schèmes, comme celui du chemin, qui explicite les séquences des voyages, ou par ce que Charles Delattre qualifie de métaphores botaniques, géométriques ou du tissage ${ }^{8}$. L'usage des schèmes nous permet d'aborder maintenant plus directement les pratiques généalogiques, fondées sur la schème de la parenté.

Evangélos Moutsopoulos relève la nécessité de recourir aux structures de parenté pour analyser les généalogies dans la mythologie grecque, qui concourent à la rationalisation de l'univers en utilisant les principes d'identité et de causalité. Or, c'est bien par le biais d'une structure de parenté que Mihaïl Nasta aborde les Éhées du catalogue de femmes pseudo-hésiodique. L'analyse de l'expression homérique è boïè révèle, en plus d'un effet d'emphase, l'évocation du passé à l'intérieur des récits enchâssés. L'auteur y repère ensuite, à travers une filiation matrilinéaire, des lieux de la tradition orale selon un encadrement métrico-narratif et des motivations propre à l'éloge, qui aident à agencer des filiations, des intrigues et leur signifiance parmi les péripéties d'une histoire fabuleuse. Ces éléments qui régissent tant les affinités de couplage des divinités que l'émergence des histoires héroïques laissent aussi apparaître les aspects eschatologiques du crépuscule des demi-dieux.

Jacques Boulogne rappelle l'existence, dans le prélude de la Théogonie, de onze séquences en hexamètres se présentant sous la forme d'un catalogue de figures divines, qui ne dévoile pas les liens entre elles. En utilisant une lecture rétrograde de ce passage qui part du telos (premier catalogue) et arrive à l'archè (troisième catalogue), il fait prévaloir à la fois l'action de grâce du poète (en fonction de trois topologies des Muses : Piérie, Olympe, Hélicon) et la possibilité de redresser le catalogue généalogique - sans prétendre relever quelque pratique mythographique. Par ce redressement du discours catalogique, il établit une structure hiérarchique à six niveaux, attachés à des références mythologiques. En combinant le déploiement généalogique avec la hiérarchie, l'auteur pratique un redressement du discours catalogique qui

\footnotetext{
${ }^{8}$ L'usage du schème du tissage peut être illustré par le Politique de Platon. Voir mon étude « Le paradigme platonicien du tissage comme modèle politique d'une société complexe », Revue de philosophie ancienne, 13 (1995), p. 108-162, où cependant je n'avais pas encore discerné le lien entre la suite des procédés de provenance d'un vêtement à partir de la laine du mouton jusqu'à la fabrication du fil et au tissage, et le procédé catalogique, comme je viens de le faire dans mon livre La proximité et la question de la souffrance bumaine, Bruxelles, Ousia, 2005, p. 675 sq. Cf. G. NAGY, Plato's Rhapsody and Homer's Music, Washigton / Athènes, 2002, p. 70 sq.
} 
assure une plus grande intelligibilité aux textes. Cette démarche a le mérite de supposer deux logiques fort différentes : celle de la narrativité et celle de la structure que celle-ci recouvre, avec la difficulté de savoir s'il n'y a pas des cas où la seconde logique se laisse éclipser par la rencontre entre, d'une part, la généalogie et, d'autre part, le devenir ou encore l'histoire.

Sur le plan de la logique narrative, Giovanni Tosetti propose une méthode historico-religieuse et sémio-narrative. Il montre comment des logographes (Acousilaos d'Argos, Hécatée de Milet, Hellanicos de Lesbos, Phérécyde d'Athènes et le Pseudo-Apollodore de la Bibliothèque) envisagent la génération des héros de la guerre de Troie et de leurs épigones comme finissant les successions généalogiques hésiodiques, concluant les récits généalogiques, en marquant la fin du temps des héros, auquel succéderait un régime temporel différent, correspondant au présent. Le temps généalogique raconté rejoint des généalogies royales, comme dans le cas de Codros. Tout se passe ainsi comme si, à une protohistoire (où règne une plus grande proximité entre les dieux et les hommes), succédait l'histoire.

La proximité entre généalogie et histoire est défendue par Emilio Suárez de la Torre. Plus en effet on s'éloigne des généalogies divines, plus la réalité anthropogonique s'impose par la transmission du sperma. Dans le cas de Pindare, qui lie les victoires sportives avec le passé mythique, la généalogie joue le rôle d'un intermédiaire qui renforce la gloire conférée par les victoires, qui ne sont pas étrangères aux origines du héros. D'où l'importance aussi du culte du dieu du lignage ou du héros colonisateur, ce qui ajoute au lien généalogique un lien religieux.

Avec cette série de communications qui affrontent les pratiques généalogiques, nous touchons à un point décisif de la thématique du colloque. On découvre deux situations opposées et extrêmes : l'une qui, proche des généalogies humaines, rapproche la généalogie et l'histoire, l'autre qui, relatant les généalogies divines, se tient sur le plan purement mythique. Entre les deux, comme tente de le faire Giovanni Tosetti, se tient une position qui marque ces deux situations selon une rupture : celle du temps raconté et du temps réel. Toutes ces approches soulèvent d'innombrables questions, qu'il faudra poser à l'avenir.

Dès lors que l'on prend au sérieux la nécessité d'un redressement du discours catalogique, et notamment de certaines formes généalogiques, nous pénétrons dans le domaine complexe et mouvant des modes d'interprétation, par des décodifications, des allégories, etc. de divers textes transmis par la tradition, parfois sous des formes réduites et esquissées du discours catalogique. C'est dans ce domaine que la mythographie révèle toute sa fécondité. Celle-ci trouverait sa source dans le texte pseudo-hésiodique des Catalogues de femmes, mais s'affirmerait surtout à partir de l'époque hellénistique.

La communication de Jacqueline Fabre-Serris constitue une bonne transition entre ce qui précède et la pratique mythographique, dans la mesure où la pensée romaine au $\mathrm{I}^{\text {er }}$ siècle avant notre ère en est arrivée à disqualifier la généalogie, non plus seulement à cause des incohérences que produit sa 
lecture littérale, mais aussi parce qu'elle a été manipulée pour la promotion de personnages politiques. L'auteur analyse cette sorte de distorsion perverse à travers la crise de rupture avec le passé, en prenant conscience des dangers pour la religion traditionnelle. Les dangers sont dus à plusieurs causes : les théologies évhéméristes des poètes et abstraites des philosophes (Mucius Scaevola); la manipulation des généalogies qui, au nom de considérations politiques, lient les hommes à des dieux ou aux événements cosmiques (Varron); les rapports entre généalogies et cultes, soumis à une argumentation pertinente (Cicéron), voire une analyse des figures de Dionysos dans une constellation divine, liée à formation des cultes au cours du temps et de la constitution d'une nouvelle «famille divine » (Diodore); une réhabilitation des catalogues généalogiques comme fondement de la divinisation (Ovide), etc. Bien que la mythographie soit un danger pour la religion nationale, elle a néanmoins contribué à la mise en forme de la culture passée et comme référence argumentative pour justifier de nouvelles visions culturelles et cultuelles. Nous découvrons ainsi l'usage du discours catalogique épuré, marqué par une structure limitée qui préserve quelque chose du passé, sans pour autant abandonner ses prétentions fonctionnelles.

Ces deux perspectives sont bien appréhendées dans leurs origines par Robert Fowler. Il envisage la mythographie comme un genre littéraire qui n'est pas lié naturellement à la mythologie, dans la mesure où celle-ci lui serait plutôt une sorte d'enfant adoptif. L'axe catalogique est ainsi détaché de l'axe mythologique. Le point de comparaison de l'ancienne mythographie doit être ainsi, d'une part, le Catalogue des femmes et, d'autre part, l'historiographie. C'est pourquoi l'auteur analyse le style narratif, en se fondant sur trois procédés : la narratologie (O. de Jong et S. Richardson), la dimension énonciative de la poésie archä̈que, notamment l'usage de la forme impersonnelle (Cl. Calame), et surtout les registres linguistiques (A. Willi et D. Biber) qu'il illustre par cinq auteurs: Hésiode, Pindare, Phérécyde, Apollonios de Rhodes et Apollodore. Pauvre de parole et de discours, appauvrie aussi par l'usage des signes et de la fable, la mythographie intègre un type de rythme qui est celui du sommaire différent de celui de la poésie. Elle s'organise aussi en dehors d'une mimèsis, des motivations, des caractères et des affects, utilisés par la poésie (épopée, tragédie, comédie...). Enfin une mise en ordre y est impliquée, où se manifeste à la fois la pratique généalogique et ce que Ricoeur appelle la mise en intrigue, que R. Fowler repère dans l'Odyssée IXXII. Dès lors que la distinction entre mythe et histoire est placée après Thucydide, la mythographie contribue à l'émergence d'un nouveau monde à l'époque hellénistique, où l'on trouve plusieurs types d'usage.

Les explications de J. Fabre-Serris et de R. Fowler ouvrent à deux thèmes majeurs: le genre de l'epitomè selon Épicure et la masse d'informations recelées dans un passage ou un texte concis. En optant pour des incisions dans un texte complexe, Épicure aide le lecteur à mieux suivre son articulation et à préserver la mémoire des lignes directrices. Face à la complexité du réel, l'homme circonscrit les choses par des configurations. Et comme l'a montré 
Ricœur, la mise en intrigue narrative forme également une configuration entre une préfiguration (la réalité) et une refiguration (la réception par l'auditeur du message $)^{9}$. En réalité, les affects sont tributaires de la façon d'arranger les matériaux du langage qui forment le mythos $^{10}$. Manifestement, la mythographie retient du « mythe » les informations minimales requises pour sauvegarder une tradition, en pratiquant un type de discours catalogique pouvant intégrer des généalogies, via le schème de la parenté. À l'opposé, la narration peut inclure une information riche. Cependant, ce ne sont pas seulement des textes longs et variables qui renferment beaucoup d'information. R. Fowler le montre en utilisant un vers d'Hésiode (fr. 41) et J. Fabre-Serris s'appuie sur des références mythographiques ponctuelles, qui l'aident à dessiner une bonne partie de la structure de la théologie politique de la cité romaine. En réalité, même un proverbe peut inclure des informations riches, et comme on l'a vu, de même des images ou des inscriptions. Seulement ces informations ne sont pas audibles ou visibles immédiatement, mais requièrent un travail de l'imagination et de la pensée pour les découvrir, tout comme il faut la médiation de la pensée pour redresser un catalogue.

Tous ces éléments montrent suffisamment la fécondité de ce genre catalogique que sont les mythographies, et que la communication de Françoise Graziani met en évidence grâce à leur rôle décisif à l'époque de la Renaissance. L'intérêt de ce travail tient à l'étude des pratiques mythographiques chez divers auteurs de l'époque (Boccace, Gyraldi, Heyne, Pictor, Cartari, etc.), et à l'organisation généalogique produite à partir de la collecte des « reliques» (éléments mythologiques, doxographiques, historiques, cosmographiques) pour réaliser une vue d'ensemble (synopsis). Nous rencontrons ainsi une démarche inverse de celle des généalogies archaïques, où la généalogie était formée par un démembrement du réel, en vue d'être reconstitué après coup. Ici, la remontée du temps n'est pas une quête de la structure du présent (ordre divin ou présence massive des morts dans l'Hadès), mais une archéologie de ce à quoi on n'a plus accès. Si bien que, dans la recomposition des mythes par un système généalogique, "l'arbitraire est ouvertement revendiqué ». Or, comme ce recours au passé vise également, selon Boccace, à "couvrir d'un merveilleux mystère les choses de la nature " (Genealogia deorum I, Proemium I, 40-46), à l'instar de ce que proposera aussi Pléthon au début de ses Lois, les distorsions généalogiques pourraient requérir quelque redressement. F. Graziani relève ici la difficulté du problème à travers les termes synopsis, syngraphè, synthesis, systasis, syntagma, qui subvertissent ce qui ne serait qu'un katalogon remémorant des noms, en ajoutant une organisation signifiante à ce qui est juxtaposé dans une discontinuité.

Par cette " composition », nous assistons à la constitution de l'association de Saturne (qui engendre les quatre éléments du monde) et de Janus, sources

\footnotetext{
${ }^{9}$ Cf. La proximité et la question de la souffrance bumaine, o.c. (n. 8), p. 62 sq.

${ }^{10}$ Voir mon texte ci-dessous.
} 
communes de l'histoire des dieux et celle des hommes; au façonnement de la figure divine de Demogorgon, inventée par Boccace pour faire prévaloir un état pré-originaire dans l'antre de l'Éternité (Boccace); et à une série de divinités chtoniennes (Pictor). Sans oublier la promotion d'un centre topologique autour de l'Arcadie. Jusqu'à quel point le recours au passé sert-il une archaiologia stricte ou requiert-il une distinction entre généalogie des dieux et celle des hommes avec comme limite celle des héros? La réponse reste ouverte. Le fait aussi que Boccace recourt, en parallèle avec Saturne, à « la métaphore de la navigation, fiction poétique qui rythme de livre en livre sa traversée des temps mythiques », soulève une question qui demeure encore en suspens dans nos études : le rôle du schème du chemin dans le discours catalogique, dont la Généalogie des dieux est une illustration parmi d'autres.

En terminant, arrêtons-nous sur la contribution d'Ezio Pellizer qui a présenté le projet du « Groupe de Recherche sur le Mythe et la Mythographie de l'Université de Trieste » pour la mise au point du Dictionnaire Étymologique de la Mythologie grecque. On Line (DEMG.OL), Il s'agit d'une mise à jour des dictionnaires de A. Carnoy (1957) et de A. Room (1983), afin de constituer une banque de données multilingue consultable en ligne. À mes yeux, ce travail ne forme pas seulement un instrument utile, mais constitue un témoignage vivant de la transmutation de la pratique séquentielle de l'écriture aux pratiques sérielles et séquentielles contemporaines, favorisées par une technologie sans précédent. Il demeure, dans l'esprit, analogue à la pratique archaïque, à savoir la capacité d'un redressement d'un système sériel et séquentiel en un monde en relief selon des formes multiples d'unité.

Université libre de Bruxelles

Centre de philosophie ancienne - CP 175/1

50, avenue Franklin Roosevelt

B - 1000 BRUXELLES

Courriel : Lambros.Couloubaritsis@ulb.ac.be 